\title{
Mandala Coloring as a Therapeutic Tool in Treating Stress-Anxiety- Depression Syndrome
}

\author{
Guo-Hui Xie a,", Qi Wang ${ }^{\text {b }}$ \\ a Early Years Research Association of Singapore. \\ b Crossland Education and Enrichment School, Singapore. \\ *Corresponding author Ph. No: 01707219556; Email: xquohui62@gmail.com \\ DOI: https://doi.org/10.54392/ajir2144 \\ Received: 21-09-2021; Revised: 18-10-2021; Accepted: 21-10-2021 Published: 28-10-2021
}

\begin{abstract}
Mandalas (in Sanskrit refers to "circle" or "discoid object") have been exclusively a part of the Eastern religions such as Hinduism, Buddhism, Taoism, Jainism and Shintoism, for hundreds of years. They represent the different aspects of the universe. They are also used as sacred meditation tools as well as consecrated symbols of prayer, most notably in China, Japan, and Tibet. Only in recent years that mandalas have been found to promote the mental as well as physical well-being or wellness, especially for those who are experiencing stress, anxiety and depression (also known as SAD syndrome). They are eventually incorporated into art as therapy and counseling as part of the repertoire of intervention tools. Generally, mandala art therapy can be divided into three different forms: (i) mandala meditation, (ii) mandala drawing, and (iii) mandala coloring. Each of these forms is a therapeutic tool that serves to help a person to relax and be at peace with oneself. According to Jungian concept of a mandala, it refers to the psychological expression of the totality of the self, and hence, mandala art therapy in whichever of its three forms can help to establish the positive wholesomeness of self. In this paper, the authors have chosen to focus on mandala coloring as a therapeutic tool and introduced the simple five-step procedure to implement it.
\end{abstract}

Keywords: Anxiety, Coloring, Mandala, Stress, Therapy.

\section{Introduction}

According to the Swiss psychiatrist, Carl Gustav Jung (b.1875-d.1961), a mandala (see Figure 1 for an example below) in its geometric configuration of signs is an oneirological symbol that represents a dreamer's search for completeness and self-unity (see Jung, 1972/73, for detail). In the eastern religions such as Buddhism, Hinduism, Jainism and Shintoism, it symbolizes the universe where we live in. Mandalas are believed to represent different aspects of the universe and they have been used as sacred tools of meditation as well as sacred symbols of prayer, most notably in China, Japan, and Tibet (see Andrea \& Neel, 2011, for detail).

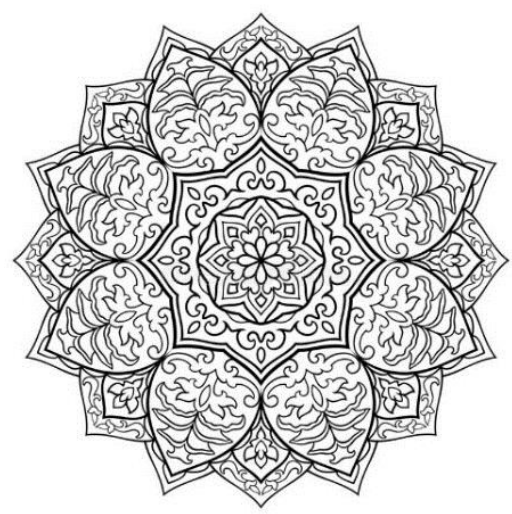

Figure 1. A Mandala 
The term mandala comes from the ancient Sanskrit word signifying "circle." However, the meaning of mandala goes beyond its simple circular shape. The symbol of mandala represents the wholeness of life, and the inter-connection that co-exists among living beings and the world in which humankind exists (Noor et al., 2017). Across a variety of cultures and within many spiritual traditions, the mandala can be found all around and takes the form of a pattern that has a circle within a circle within a circle like fractals.

Mandalas have been exclusively a part of Asian religions for hundreds of years, and for instance, Tibetan Buddhism and Hinduism feature some of the most ornate and magnificent designs. While there are tens of thousands of mandalas with unique meanings for their interpretation, perhaps the best-known mandala design outside of India is the Sri Yantra (also known as the "queen of yantras" or rajayantra), which, in Vedic traditions, represents the universe and the great divine mother or feminine principle (or the body of the goddess) of shakti or energy, power and creativity (see Daniélou, 2001, for detail). Technically speaking, Sri Yantra (see Figure 2 for an example) is not a mandala, but rather a yantra, which is the name for similar sacred geometry symbols without the iconic circular layout.

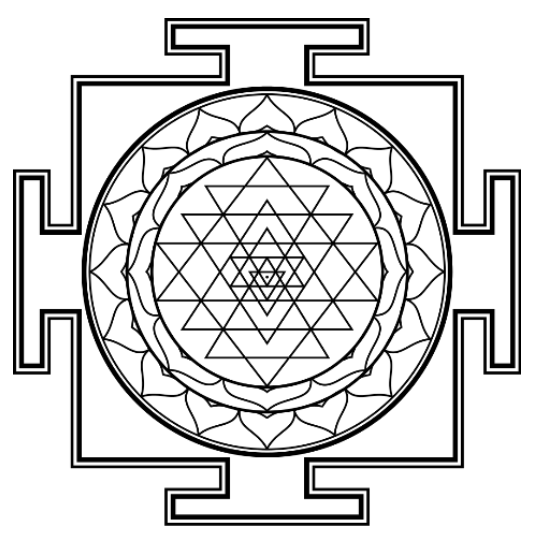

Figure 2. A Yantra

Within the two-dimensional Sri Yantra, there is a square surrounding a circle and at the heart of the circle is a lotus. Within the lotus, there are nine interconnected triangles, which represent the cosmos and the human body, that surround a central point known as a bindu. Because of its nine triangles, Shri Yantra is also known as the Navayoni Chakra because of its nine triangles (Shankaranarayanan, 1979). These nine triangles constitute a total of 43 smaller triangles. The symbolic meaning of the yantra is twofold: (i) non-duality; and (ii) reconciliation of opposites. A three -dimensional Shri Yantra is known as a Mahameru.

In this paper, the authors' main focus is on mandalas and how they can be used in the arts as therapy.

\section{Mandala Art Therapy}

When mandala is incorporated into art therapy, it becomes a form of psychotherapy in which a trained therapist uses geometric patterns to guide a client through a variety of artistic processes in an attempt to seek or restore a sense of healthy mental balance, which is known as syzygy. Art as therapy has been practiced long before the term art therapy itself was even coined. Mental health practitioners such as counselors and psychotherapists have been quite aware and understood the power of artistic expression in addressing a wide range of psychosocialemotional issues. Today, mandala applied in art as therapy (or mandala art therapy in short) is used to treat stress, anxiety and depression (also known as SAD syndrome by triangulating the three conditions) (Liu, 2021), traumarelated challenges (Nanda et al., 2010), grief or mourning (Coar, 2010; Day, 2017), anger management (Horvath, 2001), and many others. Mandala art therapy has begun to gain some attention and a gradual recognition as a therapeutic tool.

Mandala art therapy can be divided into three different forms (Noor et al., 2017), i.e., meditation, drawing or painting, and coloring. Each of these forms is briefly described as follows:

(i) Mandala meditation (Beaucaire, 2012; Dahlke, 2001; Stalin \& Joy, 2018): Also known as mandala mindfulness (Beckwith, 2014), Dankly and Stanton (2014) have described the term mindfulness as giving 
attention in a specific manner. In this form of attention, it requires a person to put his/her attention on purpose. When the tangible mandala and the intangible mindfulness are brought together, the imagery involved in the activity is seen a form of meditation, and hence, mandala meditation is a form of visual meditation. In the case of mandala meditation, the person doing it does not need to close his/her eyes to meditate. The person rests his/her gaze on the mandala that $\mathrm{s} /$ he is creating, allowing his/her thoughts that run through the mind to empty out all onto the mandala flower that is being created in agradual process.

(ii) Mandala drawing tasks (Couch, 1997; Liu et al., 2020; Smitheman-Brown \& Church, 1996): Also known as mandala painting, mandala drawing can be described as a process of drawing or painting a circle that gradually turns into a complete mandala flower like what one sees a pattern when peeking into a kaleidoscope. However, this form of mandala art therapy has already gone far beyond that. The key in the mandala drawing task is to free one's mind and intention in mandala drawing to create the patterns that narrate one's story or feelings. The intangible mind understands patterns! The mandala drawing is not just about creating a perfect circle with a repeated pattern of signs. It is also about creating a deeper connection and understanding of one's inner feelings. According to Buchalter (2013), people who are involved in mandala drawing or painting have been found to be better in expressing their feelings, thoughts, hopes, fear, and dreams. At the same time, their level of stress and anxiety also decreases; and

(iii) Mandala coloring activities (Carsley, Heath, \& Fajnerova, 2015; Curry \& Kasser, 2005; Duong, Stargell, \& Mauk, 2018): Mandala coloring is exactly what it says, i.e., the process of coloring mandalas. Coloring mandalas involves hand-eye coordination in terms of repetitive and rhythmic hand movements and focused attention. The activity allows a person to escape troubling or disturbing thoughts. It takes the person away from his/her hyperactive mind by creating a sense of safety, control and familiarity within, three things that are quite opposite to what someone feels when s/he is stressed, helpless and lost. According to Carsley and Heath (2018), mandala coloring activities have been found to be associated with decreases in test anxiety and increases in mindfulness. Educators have been recommended to incorporate free drawing/coloring activities in their classrooms for students. Carsley and Health (2018) argued that "[A]lthough structured coloring of mandalas has been suggested to be a mindfulness-based art activity for test anxiety throughout the media, free drawing and coloring may well provide the same benefits; therefore, educators can include free coloring activities within their classrooms-which are easily accessible-before a test for students who may experience test anxiety" (p. 7).

Several studies (e.g., Liu, 2021; Liu et al, 2020; Henderson, Rosen, \& Mascaro, 2007) have found the benefits of mandala art therapy though these benefits do not relate exactly to mandalas per se, but rather, more importantly, the process of applying the mandala as art therapy for stress, anxiety and depression (see Liu, 2012, for detail). Here is a list of benefits from mandala art therapy:

(1) Promote mindfulness and healing in an individual: Mandala making is one way to practice mindfulness, which can be defined as a reflective state of being aware of the present moment (see Campenni \& Hartman, 2020, for detail). In fact, Roquet and Sas (2021) did a study to show that a mindfulness-based brain-computer interface could help to augment mandala coloring for individuals with depression. Often people who practice mindfulness use quiet breathing techniques, walking or doing yoga. Mandala coloring activity is also found to promote mindfulness and through it healing takes place subconsciously (Mantzios \& Giannou, 2018).

(2) Increase a person's focus: Mandala art therapy has been found to improve the attention span and decisionmaking as well as a reduction in impulsive behaviors in children with attention deficit-hyperactivity disorder (ADHD) (Smitheman-Brown \& Church, 1996). Henderson et al. (2007) examined the benefits of the healing nature of mandalas and reported of the calming effect that mandala therapy produces on the patients while also facilitates psychic integration.

(3) Release emotion, fear, worry, tension, anxiety and stress: According to Cornell (1994), creating a mandala provides a "calming and relaxing effect on the mind and body" (p. 2). For example, a reduction in anxiety among pregnant women in their third trimester before delivery through mandala drawing and coloring (Amelia, Satiadarmma, \& Wati, 2020), post-secondary and tertiary students in their undergraduate studies through mandala coloring (Angellim, Satiadarma, \& Subroto, 2020; Noor et al., 2017); 
(4) Increase one's understanding of anxiety disorder and raise one's awareness of the condition: Through mandala coloring activities, a greater reduction in self-reported anxiety disorder than coloring a plaid design or free coloring (van der Vennet and Serice, 2012). By raising one's awareness of the anxiety condition, it causes the anxiety to lose its grip when the person clears his/her mind of worry and bring his/her own awareness back to the present.

(5) Stimulate creativity: According to Kellogg $(1977,1978)$, mandala drawing and/or coloring can offer a channel for creativity. Holbrook and Comer (2017) found that mandala could help students explore factors (e.g., moods, context) that affect their creativity through self-expression and self-reflection.

(6) Release emotional blockages and ease in socialization: Mandala art therapy has helped to improve social interaction skills in an individual with autism spectrum disorder (ASD) (Kim \& Ong, 2018);

(7) Decrease impulsiveness: According to Smitheman-Brown and Church (1996), doing mandala exercises produces the effect of increasing attentional abilities, while over a period of time, also decreasing impulsive behaviors. This is also reported by John et al. (2013) to the American Art Therapy Association Research Committee. Mandala activities serve to promote "better decision making, completion of task, general growth in developmental level, and an interest in personal aesthetics" (Smitheman-Brown \& Church, 1996, p. 252).

(8) Promote self-discovery: Odle (1998) reported that mandala is a good tool for spiritual healing and selfdiscovery. Mahar, Iwasiw and Evans (2012) also found that mandala drawing and coloring activities were fun and enjoyable, allowing the participants to express themselves freely. Through the process, the participants were able to describe experiences of self-discovery (Mahar, Iwasiw, \& Evans, 2012).

\section{Application of Mandala Coloring as a Therapeutic Tool}

In this paper, the authors have chosen to focus on mandala coloring as a therapeutic tool, which Mozdzierz, Lisiecki and Macchitelli (1989) and Goforth (2001) described as powerful and ubiquitous encouragenic in art therapy. The authors have used this as a form of no-talk therapy (see Straus, 1999, for detail) in counseling with their clients, especially those who experience stress, anxiety and the early stage of depression (mild to moderate level of severity).

To begin with, the mandala coloring activity as a therapeutic tool has been reported by Dodd (2004) to help a person to relax and be at peace with oneself. This is the beginning of helping oneself or others to move toward both mental and physical wellness (Fincher, 2000). There are many off-the-shelf mandala coloring books that are readily available for purchase and use by buyers who want to do the activity on their own without a therapist's facilitation. Generally, these mandala coloring books are easy to color and the symmetry of each mandala design offers a satisfying end result after it is colored. The coloring can help to create a beautiful mandala and here are five key stress-free steps to follow:

Step \#1. Always begin with some idea about the color scheme one has in mind. One can also select the colors of one's preference. Coloring mandalas is all about relaxation and focus, but exaggerating the color themes is not the way to go. From a mandala therapist's perspective, a person who does mandala coloring should try and limit the colors, preferably three to five. By doing so, the colored mandala will look more attractive than one with multiple colors. Most importantly, whatever color scheme one has already chosen, it is important to stick to one's selected theme.

Step \#2. Be systematic or organized in the mandala coloring routine. The center of the mandala is always the starting point because the center is what provides a person the whole view of what $\mathrm{s} /$ he sees. Hence, this is the main reason to start coloring from the center and then gradually work outward. In this way, the person coloring the mandala will soon create an attractive and symmetrical piece.

Step \#3. It would be good, if not better, always to stay meticulous using only the same selected colors for any repeating elements in the mandala coloring. This is because, by doing so, one can ensure that the mandala being colored remains symmetrical and balanced, and the end result is that the finished mandala will be an interesting piece to look at in awe and wonder. When creating the colored pattern, anyone can easily observe and tell which parts are repeating and which ones are not. Though the coloring procedure to be tedious, the effort is worth every 
move. This is the first step that will lead one to a more detailed mandala as one progresses in mandala coloring routine.

Step \#4. It is very important to relax or free the mind from any other thoughts. The authors have encountered many, if not all, beginners feeling tense in mandala coloring, i.e., especially when it is his/her first attempt. However, one should be aware that tension experienced in the mandala coloring activity will lead the person to distraction. His/her mind will soon wander back to the unpleasant experience of feeling stressed and anxious. Hence, failure to stay focused on the coloring activity only leads to a more strained and tensed effort in executing the task. Hence, during the mandala coloring activity, one should ensure that $\mathrm{s} /$ he does not overthink. It is important for the person to let the mind go free and be immersed in the coloring activity. It is good to let one's creativity to glow in the page of mandala coloring. Soon, in the process of coloring the mandalas, both stress and anxiety just fade away in a gradual manner.

Step \#5. The fifth and last step in mandala coloring is to add tonal gradients and blends. Colors often speak volumes when they are used in creating an impressive mandala. Each color goes with a certain chakra (Leadbeater, 2013), which is an energy force believed to link to one's body and the immediate environment, and supposedly to help with one's well-being (Fincher, 2000). Mandala therapists often make claims that mandalas are so good for one's well-being because they have chakras in them (Leadbeater, 2013, for detail). However, there is still much a person can do with the colors of his/her choice to achieve the results s/he wants: The tonal gradients and blends should be his/her first task to attempt if s/he is seeking for something striking.

\section{Conclusion}

In this paper, the authors have reported that using a mandala coloring as a form of healing is beneficial for both children and adults who are experiencing stress, anxiety and/or early stage of depression. It allows their unsettling or disturbing thoughts in the mind to escape onto a piece of paper. Instead of hoarding all of these unpleasant thoughts and anxious feelings inside, they can now put them on a piece of paper. The mandala coloring activity instantly frees the mind and body of stress, anxiety and depression (or SAD syndrome for short), decreasing these three SAD symptoms that have plagued them all the while.

Mandalas, therefore, provide some kind of meditation even through drawing and coloring tasks to help one to focus on one's attention. These symmetrical circles can bring harmony through their shapes and colors transforming negative thoughts and feelings into positive ones, and hence, bring about balance to one's life. In other words, a mandala, according to Jung (1972/73), "is the psychological expression of the totality of the self" (p. 20).

Finally, mandala coloring also gives a person a better understanding of what is going on in his/her mind so that in the process of coloring the mandalas (whether or not under a therapist's facilitation or guidance), each of them can then find the proper tips, tools and techniques that work best for him/her.

\section{References}

Amelia, G., Satiadarma, M.P., Wati, L., (2020), The effects of drawing and coloring mandala on anxiety among pregnant women in their third trimester, Advances in Social sciences, Education \& Humanities Research, 478, 997-1002. https://dx.doi.org/10.2991/assehr.k.201209.158

Andrea, A. J., Neel, C., (2011), World history encyclopedia, ABC-CLIO, Santa Barbara, California.

Angellim, C., Satiadarma, M.P., Subroto, U., (2020), Mandala drawing for reducing test anxiety in college student, Proceedings of the 2nd Tarumanagara International Conference on the Applications of Social Sciences and Humanities (TICASH 2020), Advances in Social Science, Education and Humanities Research, Atlantis Press, 478, 451-456. https://dx.doi.org/10.2991/assehr.k.201209.069

Beaucaire, M., (2012), The art of mandala meditation: Mandala designs to heal your mind, body and spirit, Simon and Schuster, New York.

Beckwith, P., (2014), Mindfulness and mandalas: Alternative therapeutic techniques for AOD adolescents, Capital University Undergraduate Research Journal, 1-5.

Buchalter, S., (2013), Mandala symbolism and techniques: Innovative approaches for professionals, Jessica Kingsley Publishers, Philadelphia. 
Campenni, C.E., Hartman, A., (2020), The effects of completing mandalas on mood, anxiety, and state mindfulness, Art Therapy: Journal of the American Art Therapy Association, 37(1), 25-33. https://doi.org/10.1080/07421656.2019.1669980

Carsley, D., Heath, N.L., (2018), Evaluating the effectiveness of a mindfulness coloring activity for test anxiety in children, The Journal of Educational Research, 112(2), $143-151$. https://doi.org/10.1080/00220671.2018.1448749

Carsley, D., Heath, N.L., Fajnerova, S., (2015), Effectiveness of a classroom mindfulness coloring activity for test anxiety in children, Journal of Applied School Psychology, 31(3), 239-255. https://doi.org/10.1080/15377903.2015.1056925

Coar, H., (2010), Using mandalas to support grieving female adolescents: A case study, California State University Press, Long Beach, USA.

Cornell, J., (1994), Mandala, Quest Books, Wheaton, IL.

Couch, J.B., (1997)., Behind the veil: Mandala drawings by dementia patients, Art Therapy: Journal of the American Art Therapy Association, 14(3), 187-193. https://doi.org/10.1080/07421656.1987.10759280

Curry, N.A., Kasser, T., (2005), Can coloring mandalas reduce anxiety?, Art Therapy: Journal of the American Art Therapy Association, 22(2), 81-85. https://doi.org/10.1080/07421656.2005.10129441

Dahlke, R., (2001), Mandalas for meditation, Sterling Publishing Company, Inc, New York.

Daniélou, A., (2001), The Hindu temple: Deification of eroticism, Inner Traditions/Bear \& Company, Rochester, New York.

Dankly, C., Stanton, M., (2014), Teaching clients to use mindfulness: A practical guide, Routledge, New York.

Day, J., (2017), Mandala making through crochet to navigate grief. In B. MacWilliam (Ed.), Complicated grief, attachment, and art therapy: Theory, treatment, and 14 ready-to-use protocols, Jessica Kingsley, London.

Dodd, J. L., (2004). The use of the mandala to promote relaxation with children in a partial hospitalization program, Ursuline College, Cleveland.

Duong, K., Stargell, N.A., Mauk, G.W., (2018), Effectiveness of coloring mandala designs to reduce anxiety in graduate counseling students, Journal of Creativity in Mental Health, 13(3), 318-330. https://doi.org/10.1080/15401383.2018.1437001

Fincher S.F., (2000), Coloring mandalas 1: For insight, healing, and self-expression, Shambhala Publications, Boston.

Goforth, M., (2001)., Mandalas in art therapy: A therapeutic tool to help children focus, Ursuline College, Cleveland, $\mathrm{OH}$.

Henderson, P., Rosen, D., Mascaro, N., (2007), Empirical study on the healing nature of mandalas, Psychology of Aesthetics, Creativity, and the Arts, 1(3), 148-154. https://doi.org/10.1037/1931-3896.1.3.148

Holbrook Jr, R. L., Comer, D.R., (2017), Mandalas: A simple project to explore creativity, Management Teaching Review, 2(3), 202-210. https://doi.org/10.1177/2379298117709782

Horvath, V.M., (2001), Mandala therapy: Investigating physiology, color, and chakras in adults with mood disorder, ProQuest Dissertation Publishing, Ann Arbor, MI, USA.

John, P. S., Bermudez-Rodriguez, D., Collie, K., Kaiser, D., Deaver, S., Orr, P., Betts D., Robb, M., Vennet, R.V., (2013), American Art Therapy Association Research Committee: Art Therapy Outcome - Bibliography, American Art Therapy Association.

Jung, C.G., (1972/1973), Mandala symbolism, Princeton University Press, United States of America.

Kellogg, J., (1977), The use of the mandala in psychological evaluation and treatment, American Journal of Art Therapy, 16(4), 123-134.

Kellogg, J., (1978), Mandala: The path of beauty, Mandala Assessment and Research Institute, Baltimore.

Kim, T.H., Ong, I.L., (2018), Mandala art therapy: Intervention for individual with autism spectrum disorder, Jurnal Psikologi Malaysia, 32(1), 97-113.

Leadbeater, C.W., (2013). The chakras.Quest Books, Theosophical Society in America, Wheaton.

Liu, C., Chen, H., Liu, C.Y., Lin, R.T., Chiou, W. K., (2020), Cooperative and individual mandala drawings have different effects on mindfulness, spirituality, and subjective well-being, Frontiers in Psychology, 11, 564430. https://doi.org/10.3389/fpsyg.2020.564430

Liu, W., (2021), A brief exploratory review of mandala drawing \& coloring in promoting mental health/well-being among young children during the Covid-19 pandemic lockdown, Early Years Research, 1(1), 32-39. 
Mahar, D.J., Iwasiw, C.L., Evans, M.K., (2012), The mandala: First-year undergraduate nursing students' learning experiences, International Journal of Nursing Education Scholarship, 9(1). https://doi.org/10.1515/1548923X.2313.

Mantzios, M., Giannou, K., (2018), When did coloring books become mindful? Exploring the effectiveness of a novel method of mindfulness-guided instructions for coloring books to increase mindfulness and decrease anxiety, Frontiers in Psychology, 9(56), 1-9 https://doi.org/10.3389/fpsyg.2018.00056

Mozdzierz, G.J., Lisiecki, J., Macchitelli, F.J., (1989), The mandala of psychotherapy: The universal use of paradox: New understanding and more confusion, Psychotherapy: Theory, Research, Practice, Training, 26(3), 383388. https://doi.org/10.1037/h0085450

Nanda, U., Barbato Gaydos, H.L., Hathorn, K.,Watkins, N., (2010), Art and post-traumatic stress: A review of the empirical literature on the therapeutic implications of artwork for war veterans with post-traumatic stress disorder, Environment and Behavior, 42(3), 376-390. https://doi.org/10.1177/0013916510361874

Noor, S.M., Saleem, T., Azmat, J., \& Arouj, K., (2017), Mandala-coloring as a therapeutic intervention for anxiety reduction in university students, Pakistani Armed Forces Medical Journal, 67(6), 904-907.

Odle, K.S., (1998), Mandala: A tool for spiritual healing and self-discovery - a personal exploration, Prescott College. Roquet, C.D., Sas, C., (2021), A Mindfulness-Based Brain-Computer Interface to Augment Mandala Coloring for Depression: Protocol for a Single-Case Experimental Design, JMIR Research Protocols, 10(1). https://doi.org/10.2196/20819

Shankaranarayanan, S., (1979), Sri Chakra, Lotus Public, India.

Smitheman-Brown, V., Church, R.P., (1996), Mandala drawing: Facilitating creative growth in children with A.D.D. or A.D.H.D, Art Therapy: Journal of the American Art Therapy Association, 13(4), 252-262. https://doi.org/10.1080/07421656.1996.10759233

Stalin, G.K., Joy, R.A.P, (2018), The healing nature of mandala magic, International Journal of Nursing Education and Research, 6(3), 281-282. http://dx.doi.org/10.5958/2454-2660.2018.00066.2

Straus, M.B., (1999), No-talk therapy for children and adolescents, WW Norton \& Company, New York.

Tanabe, W.J., (2001), Japanese mandalas: Representations of sacred geography, Japanese Journal of Religious Studies, 28(1/2), 186-188.

Van Der Vennet, R., Serice, S., (2012), Can coloring mandalas reduce anxiety? A replication study, Art Therapy: The Journal of the American Association of Art Therapists, 29(2), 87-92. https://doi.org/10.1080/07421656.2012.680047

Does this article screened for similarity: Yes

Funding: No funding was received for conducting this study.

Conflict of Interest: The authors have no conflicts of interest to declare that they are relevant to the content of this article.

\section{About The License}

(C) The author(s) 2021. The text of this article is open access and licensed under a Creative Commons Attribution 4.0 International License

\section{Cite this Article}

Guo-Hui Xie \& Qi Wang, Mandala Coloring as a Therapeutic Tool in Treating Stress-Anxiety-Depression Syndrome, Asian Journal of Interdisciplinary Research, 4(4) (2021) 30-36. https://doi.org/10.54392/ajir2144 